\title{
Bone Mineral Density Changes after Orchiectomy using a Scrotal Approach in Rats
}

\author{
Seong Jun Ryu, Dal Sung Ryu, Jong Yul Kim, Jeong Yoon Park, Kyung Hyun Kim, \\ Dong Kyu Chin, Keun Su Kim, Yong Eun Cho, Sung Uk Kuh \\ Department of Neurosurgery, The Spine and Spinal Cord Institute, Gangnam Severance Hospital, \\ Yonsei University College of Medicine, Seoul, Korea
}

\begin{abstract}
Objective: To investigate a suitable animal model for studies of male osteoporosis. Osteoporosis has a particularly high incidence in postmenopausal women, resulting in a substantial amount of research with respect to this disease in women. However, research on osteoporosis in men is still lacking.

Methods: Twenty 10-week-old male Sprague Dawley rats were used in this study, including 4 rats used to establish a baseline bone mineral density (BMD). The other 16 rats were divided into two groups: a sham surgery group $(n=8)$, which underwent a sham operation, and an orchiectomized rat group $(\mathrm{OCX})(\mathrm{n}=8)$, which underwent bilateral $\mathrm{OCX}$ at 10 weeks of age. Bone mineral density was measured in 4 rats from both the sham surgery group and the OCX group 8 weeks after the surgery, while BMD in the remainder of the rats was measured 10 weeks post-surgery.

Results: Femoral BMD at 8 weeks post-surgery was found to be significantly lower in the OCX group compared to the sham group; a finding that was also similar 10 weeks post-surgery.

Conclusion: 8 weeks after undergoing orchiectomy performed via a scrotal, white rats are a suitable model for studies of male osteoporosis.
\end{abstract}

Key Words: Animal model $\cdot$ Osteoporosis $\cdot$ Male $\cdot$ Orchiectomy $\cdot$ Bone mineral density $\cdot$ Femur

\section{INTRODUCTION}

Korean society is rapidly aging in comparison to other developd countries. In 2000, Korea became an aging population with $7.2 \%$ of the total population aged 65 or older. It has been estimated that Korea will become an aged society in 2019, and a super-aged society in $2026^{17}$. Hence, it is safe to assume that osteoporosis will become the next most important geriatric disease, following coronary artery disease (CAD) and diabetes mellitus (DM), resulting in rising costs to society for prevention and treatment.

\footnotetext{
- Received: March 12, 2015 - Revised: April 22, 2015

- Accepted: April 23, 2015

Corresponding Author: Sung Uk Kuh, MD, PhD

Department of Neurosurgery, Gangnam Severance Hospital, The Spine and Spinal Cord Institute, Yonsei University College of Medicine, 211,

Eonju-ro Gangnam-gu, Seoul 135-720, Korea

Tel: +82-2-2019-3390, Fax: +82-2-3461-9229

E-mail: kuhsu@yuhs.ac

®This is an Open Access article distributed under the terms of the Creative Commons Attribution Non-Commercial License (http://creativecommons.org/ licenses/by-nc/3.0/) which permits unrestricted non-commercial use, distribution, and reproduction in any medium, provided the original work is properly cited.
}

Research on osteoporosis has so far been primarily performed on postmenopausal women, due to the high incidence of disease in this population. However, it is important to acknowledge that $14.5 \%$ of men who underwent spinal surgery had osteoporosis, which can result in higher reported morbidity and mortality if fracture was to occur ${ }^{10)}$. This percentage is onethird of that found among women ${ }^{3)}$. For this reason, research on male osteoporosis is essential.

Regarding osteoporosis in men, it has been reported that the androgen deprivation therapy used in patients with prostate cancer is complicated by osteoporosis ${ }^{4)}$ and the high alkaline phosphatase levels in these patients ${ }^{14)}$ allows us to deduce that bone loss is occurring in these patients. It is also known that continued use of androgen deprivation therapy in patients with prostate cancer results in a worsening of osteoporosis ${ }^{5}$. In these cases, anti-resorptive drugs, such as bisphosphonate, are used in prevention. Consequently, it is very important to understand their effect. Many articles have been published regarding the effects of anti-resorptive drugs in orchiectomized animal models of male osteoporosis ${ }^{1,6,11}$. However, before the effects of these drugs are evaluated in animal models, it is also important to validate whether the animal model is already compromised by osteoporosis. 
The use of ovariectomized white rats as a female osteoporosis model, included specific procedural guidelines and disease development findings, has been reported ${ }^{15)}$. However, similar reports on male osteoporosis animal models are scarce, except for articles dealing with male osteoporosis risk factors ${ }^{16,19)}$ and histological analyses ${ }^{7}$ in orchiectomized white rats. We sought to evaluate these issues in detail, and to statistically verify changes in bone mineral density (BMD) in our research.

In this study, we focused on the verification of BMD changes over time in rats bilaterally orchiectomized via a scrotal approach (OCX), and to describe the specific procedures used to create our male osteopenia animal model.

\section{MATERIALS AND METHODS}

\section{Animals}

Twenty 10-week-old male rats purchased from Orient Bio (Gyonggi, Korea) (Sprague-Dawley, weight 210-220 g) were acclimated in a comfortable environment (temperature 22士 $4^{\circ} \mathrm{C}$, humidity 65 $\pm 5 \%$, day-night cycle 6:00 AM-6:00 PM). The environment was specific pathogen free. Two rats were housed in each stainless steel wire cage. The experimental group was randomly selected. This study was approved by the Yonsei University Health System Institutional Animal Care and Use Committee.

\section{Experimental Design}

Four 10-week-old rats (control group) were sacrificed, their femora were removed, and BMD was measured in these femora. The living 16 rats were divided into two groups. The sham operation $(\mathrm{SO})$ group $(\mathrm{n}=8)$, underwent a sham operation at 10 weeks of age, and the OCX group $(n=8)$ underwent bilateral OCX, intended to change bone mineral density, at 10 weeks of age. Eight weeks after the OCX or SO, four rats from each of the two groups were sacrificed, and their femora were obtained and BMD was measured. Ten weeks after OCX or $\mathrm{SO}$, the living rats in each group were sacrificed and the BMDs of their femora were measured. All rats were sacrificed using $\mathrm{CO}_{2}$ inhalation.

\section{Femur BMD Measurement}

Rats were euthanized at weeks 10, 18, and 20, and both femora from each rat were obtained. BMD was measured in the femora using micro computed tomography (CT): SkyScan 1173 (Bruker-CT, Kartuizersweg 3B 2550 Kontich, Belgium) and NRecon (Ver. 1.6.9.4) software (Fig. 1).

\section{Orchiectomy and Sham Operation Procedure}

Anesthesia was induced with 5\% isoflurane and Rompun (2.5 mg/kg, Bayer Korea) and Zoletil (5 mg/kg, Virbac Korea) were injected intra-peritoneally for generalized anesthesia. 2.5 $\%$ isoflurane and oxygen were administered via a coaxial nose cone for anesthesia maintenance. Bilateral orchiectomy was performed via a scrotal approach. The anesthetized rat was placed supine on the operating table and its position was fixed using sticking tape. The scrotal hair was bilaterally shaved (Fig. 2A). A betadine prep was performed as an aseptic maneuver (Fig. 2B). If the cremaster muscle was stimulated during the betadine prep, resulting in ascension of the testes, a downward stroke was performed to lower the testes back into place. A small, 1.0-cm median incision was made through the skin at the tip of the scrotum (Fig. 2C). The cremaster muscles were opened with a small, 7-mm incision. At the entrance to the scrotal cavity, the testicular fat pad was located and pulled through the incision using blunt forceps. The cauda epididymis was pulled out along with the testis, followed by the caput epididymis, the vas deferens and the testicular blood vessels (Fig. 2D). After identifying the testis, epididymis, vas deferens and testicular blood vessels, a single ligature was placed on the spermatic cord around the vas deferens and the blood vessels (Fig. 2E). The testis and epididymis were removed. This procedure was repeated for on the other testis and epididymis. The cremaster muscle and scrotal skin were sutured layer by
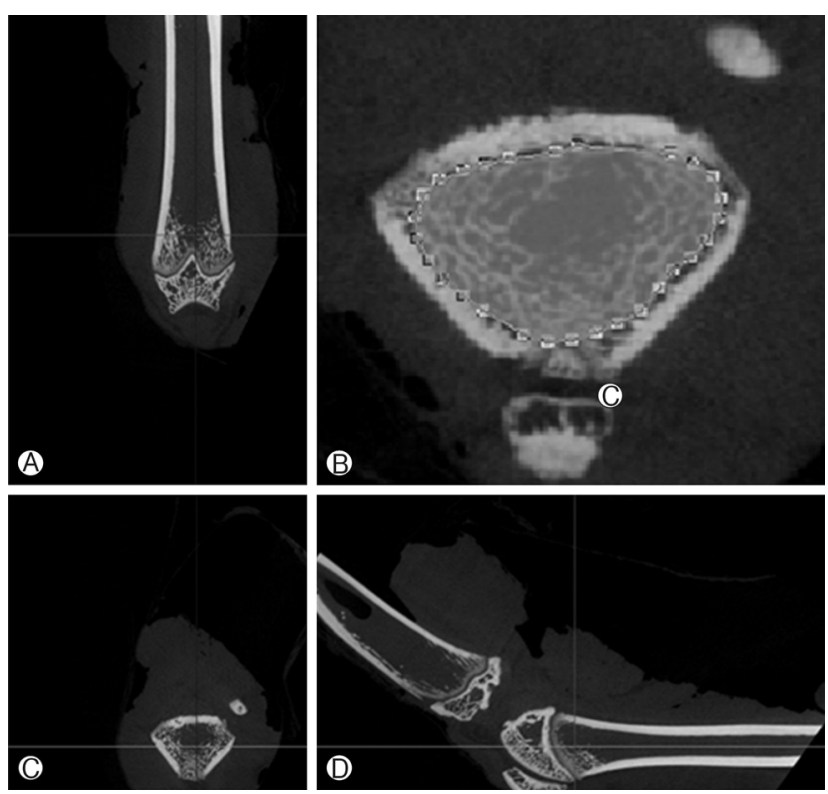

Fig. 1. BMD measurement using NRecon software. BMD was measured in the femora using micro computed tomography: SkyScan 1173 (Bruker-CT, Kartuizersweg 3B 2550 Kontich, Belgium) and NRecon (Ver. 1.6.9.4) software. 
layer (Fig. 2F).

The same preparation was performed on animals in the sham operation group, allowing the authors to visually identify the testis, epididymis, vas deferens and testicular blood vessels. After visual identification, the cremaster muscle and skin were sutured without ligation or resection.

\section{Statistical Analysis}

Statistical analysis was performed using SPSS software (version 20.0, Chicago, IL, USA). The Mann-Whitney test was used to compare values between the SO group and the OCX group, and the Wilcoxon signed-rank test was used to compare the mean values of $\mathrm{BMD}$ within the same group. Probability values $<0.05$ were considered statistical significant.

\section{RESULTS}

No procedural deaths occurred during the course of the experiment. Micro CT scan revealed male osteoporosis in orchie-
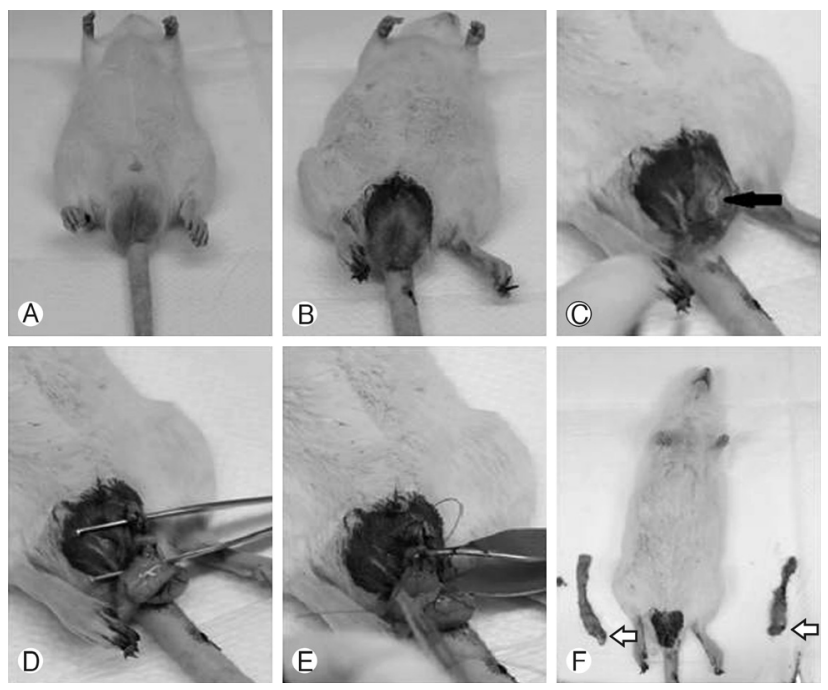

Fig. 2. Procedures for orchiectomy in rat. Anesthetized rat was laid supine on the operating table. Scrotal shaving was done (A). Betadine prep was performed as an aseptic maneuver (B). A small, 1.0- $\mathrm{cm}$ median incision was made through the skin at the tip of the scrotum (C). The cremaster muscles were opened with a small 7-mm incision. Thick black arrow: cremaster muscle. The testicular fat pad was localized and pulled through the incision using blunt forceps. The cauda epididymis was pulled out along with the testis, followed by the caput epididymis, the vas deferens and the testicular blood vessels (D). A single ligature was placed around the vas deferens and blood vessels $(\mathrm{E})$. The testis was then removed. This procedure was repeated for the other testis. Muscle and skin were sutured layer by layer $(F)$. Thick white arrow: Testis and epididymis surrounded by fat pad were totally removed. ctomy group at POD 8 weeks and POD 10 weeks. There are marked deficiencies in trabecular bone (Fig. 3). Baseline BMD was measured in 10-week-old rats (control group) and a value of $0.244 \pm 0.073 \mathrm{~g} / \mathrm{cm}^{3}$ was obtained. The mean femur BMD was significantly different between the OCX rats and the sham operation rats eight weeks after OCX $\left(0.363 \pm 0.048 \mathrm{~g} / \mathrm{cm}^{3}\right.$ vs. $0.205 \pm 0.019 \mathrm{~g} / \mathrm{cm}^{3}, \mathrm{p}=0.029$ ). The mean femur BMD was also significantly different between the OCX rats and the sham operation rats ten weeks after OCX $\left(0.451 \pm 0.076 \mathrm{~g} / \mathrm{cm}^{3}\right.$ vs. 0.280 $\pm 0.008 \mathrm{~g} / \mathrm{cm}^{3}, \mathrm{p}=0.029$ ) (Table 1 and Fig. 4). No significant differences in chronologic BMD were found in the same groups, in accordance with Wilcoxon signedranked test.

\section{DISCUSSION}

The incidence of osteoporosis is rising in concordance with the rise in the geriatric population. Hence, understanding the etiology of osteoporosis will become helpful in improving the prognosis of spinal fusion surgery in osteoporotic patients. The morbidity and mortality of fracture in male osteoporosis patients are higher than those in the female population ${ }^{10)}$, which necessitates additional research on osteoporosis in men. In the current study, we analyzed changes in $\mathrm{BMD}$ in a representative animal model (bilateral OCX rat). Having an osteoporosis animal model in which to analyze bone quality is essential for
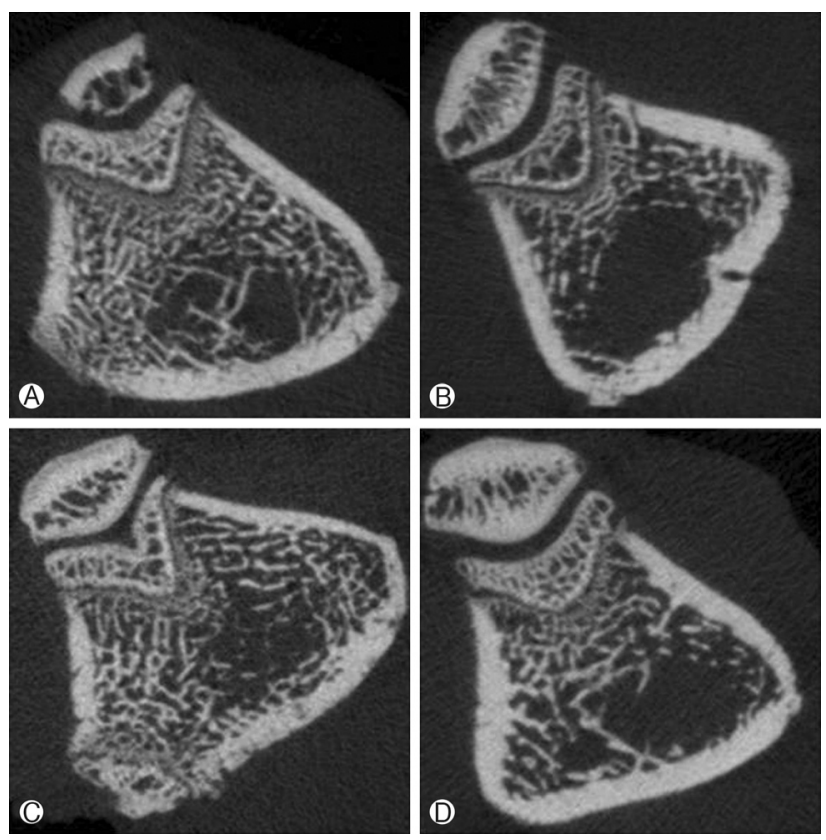

Fig. 3. Micro CT Axial images of femora. (A, C) shows Sham group rats, each rats are POD 8 weeks and POD 10 weeks. (B, D) shows OCX group rats, each rats are 8 weeks and 10 weeks. 
Table 1. The changes in $\mathrm{BMD}\left(\right.$ Mean $\left.\pm \mathrm{SD} \mathrm{g} / \mathrm{cm}^{3}\right)$ of Sham and OCX groups

\begin{tabular}{lcc}
\hline \hline \multirow{2}{*}{ Group (POD) } & \multicolumn{2}{c}{$\mathrm{BMD}\left(\mathrm{g} / \mathrm{cm}^{3}\right)$} \\
\cline { 2 - 3 } & Mean $\pm \mathrm{SD}$ & $\mathrm{p}$-value \\
\hline Sham (8 weeks) & $0.363( \pm 1.048)$ & $0.029^{*}$ \\
OCX (8 weeks) & $0.205( \pm 0.019)$ & \\
Sham (10 weeks) & $0.451( \pm 0.176)$ & $0.029^{*}$ \\
OCX (10 weeks) & $0.280( \pm 0.008)$ & \\
\hline
\end{tabular}

POD, post operation date; Sham, sham surgery group; OCX, orchiectomy group.

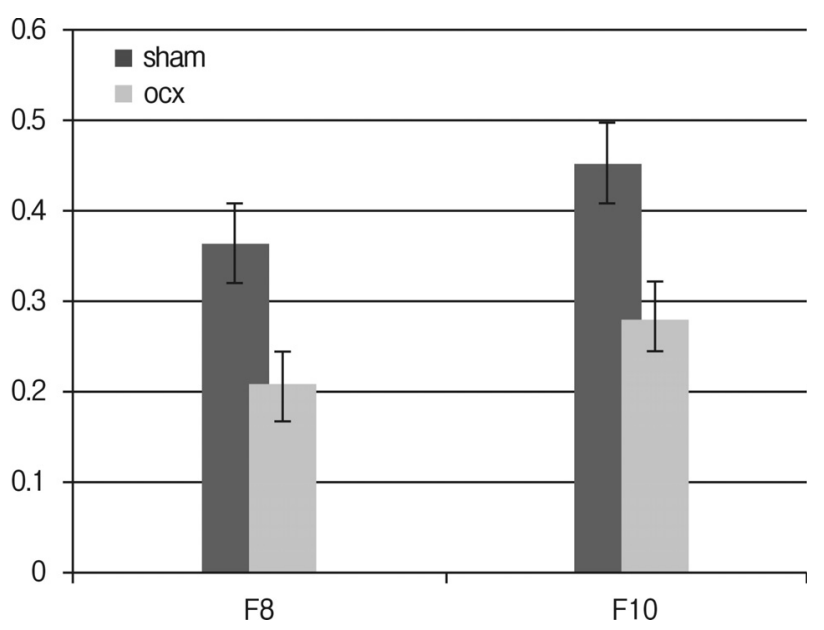

Fig. 4. The changes in $B M D\left(g / \mathrm{cm}^{3}\right)$ in the Sham and OOX groups. The measured BMD was lowest at 8 weeks, after which BMD increased.

research evaluating the effects and safety of treatments. OCX is classified as a gonadectomy which, along with ovariectomy, is a method for establishing an osteoporotic animal model ${ }^{12)}$. This method is thought to be highly relevant to and representative of prostate cancer patients with osteoporosis undergoing therapeutic androgen deprivation. There have been many articles that have used ovariectomized rats as models for osteoporosis in postmenopausal women ${ }^{13)}$. However, studies that detail the creation of precise male osteoporosis animal models and provide accompanying data on chronologic $\mathrm{BMD}$ results in these models have been lacking. Therefore, we sought to outline the procedures we used and report the resulting changes in BMD we observed in the current study.

Orchiectomy can be performed by a few different methods. Surgical methods differ in the direction of approach and location of incisions. The usual approaches are on the scrotal and abdominal sides, and the skin incisions usually made are single midline scrotal, double median scrotal and midline abdominal incisions. We performed orchiectomy in rats using double median scrotal incisions. We chose this method because the dou- ble median scrotal incision requires the shortest incision relative to the other possible single midline incisions. We also chose to use a scrotal approach because it allows for faster location of the testis, compared to an abdominal approach; thereby shortening the duration of operation. This study reports the sequential delineation of orchiectomy using double median scrotal incisions.

Previous studies have not been clear regarding the appropriate age of rats for the model or on the timing of significant $\mathrm{BMD}$ decreases after OCX when using the OCX rat model. In cases of ovariectomy, growing female rats tend to show a more sensitive skeletal response compared to aged female rats ${ }^{18)}$. If skeletally immature rats are used, a low peak bone mass is achieved. This is considered to be a high risk factor for human osteoporotic problems. This trait is why the skeletally immature rat is an appropriate animal model for research on endocrine, nutritional and environmental factors, all of which can influence peak bone mass ${ }^{12}$. We believed that growing orchiectomized rats would also show a greater response in their skeletal systems. Although rats reach sexual maturity at the age of 10 weeks, their skeleton is considered mature after 10 months ${ }^{8)}$. Because there is a time difference between endocrinologic maturation and skeletal system maturation, low peak bone mass was obtained by using 10 -week-old orchiectomized rats.

There are a few reports of decreasing bone mass starting 4 weeks after ovariectomy, which led to considerable bone mass loss at 8 and 10 weeks ${ }^{2,9)}$. Although there are studies which found decreasing bone mass after orchiectomy and reports of osteoporosis following androgen deprivation in patients with prostate cancer, studies regarding the exact timing of this decline were lacking. This study revealed the exact timing of decreasing bone quality with serial measurement using micro computed tomography. Both the 8-week and 10-week postorchiectomy groups showed statistically significant reductions in bone mass in our study. However, BMD in the 8-week group was determined to be lower. Hence if one were to conduct a study using an animal model of male osteoporosis to evaluate many bone related variables and BMD measurements, our 8week post-orchiectomy animal model would be most suited to this purpose.

Bone mass was shown to rise between weeks 8 and 10 after orchiectomy. In our study, as only 4 rats were used, the mean $\mathrm{BMD}$ we measured may not have been a precise representative of the entire rat species BMD. We believe that the increase in $\mathrm{BMD}$ observed after orchiectomy may have been due to other compensatory actions for the lack of sex hormones. Furthermore, because a histologic evaluation of osteoid numbers after OCX was not conducted in the current study, further research pursuing chemical markers and using a pathological evaluation may be required. 


\section{CONCLUSION}

Osteoporotic animal models can be easily established using bilateral orchiectomy, the details of which we have described above. Statistically significant decreases in bone mass were observed starting at 8 weeks post-operation. Bone mass increased over time even after bilateral orchiectomy, but was still statistically significant compared to the sham operation group when measured at the same time.

\section{REFERENCES}

1. Al-Shahat AR, Shaikh MA, Elmansy RA, Shehzad K, Kaimkhani ZA: Prostatic assessment in rats after bilateral orchidectomy and calcitonin treatment. Endocr Regul 45:29-36, 2011

2. Bauss F, Dempster DW: Effects of ibandronate on bone quality: preclinical studies. Bone 40:265-273, 2007

3. Chin DK, Park JY, Yoon YS, Kuh SU, Jin BH, Kim KS, et al: Prevalence of osteoporosis in patients requiring spine surgery: incidence and significance of osteoporosis in spine disease. Osteoporos Int 18:1219-1224, 2007

4. Daniell HW: Osteoporosis after orchiectomy for prostate cancer. J Urol 157:439-444, 1997

5. Daniell HW, Dunn SR, Ferguson DW, Lomas G, Niazi Z, Stratte PT: Progressive osteoporosis during androgen deprivation therapy for prostate cancer. J Urol 163:181-186, 2000

6. Gradosova I, Zivna H, Palicka V, Hubena S, Svejkovska K, Zivny P: Protective effect of atorvastatin on bone tissue in orchidectomised male albino Wistar rats. Eur J Pharmacol 679:144-150, 2012

7. Gunness M, Orwoll E: Early induction of alterations in cancellous and cortical bone histology after orchiectomy in mature rats. J Bone Miner Res 10:1735-1744, 1995

8. Jee WS, Yao W: Overview: animal models of osteopenia and osteoporosis. J Musculoskelet Neuronal Interact 1:193-207, 2001

9. Kalu DN: The ovariectomized rat model of postmenopausal bone loss. Bone Miner 15:175-191, 1991

10. Kamel HK: Male osteoporosis: new trends in diagnosis and therapy. Drugs Aging 22:741-748, 2005

11. Khedr NF, El-Ashmawy NE, El-Bahrawy HA, Haggag AA, El-Abd EE: Modulation of bone turnover in orchidectomized rats treated with raloxifene and risedronate. Fundam Clin Pharmacol 27: 526-534, 2013

12. Lelovas PP, Xanthos TT, Thoma SE, Lyritis GP, Dontas IA: The laboratory rat as an animal model for osteoporosis research. Comp Med 58:424-430, 2008

13. Miller SC, Bowman BM, Jee WS: Available animal models of osteopenia--small and large. Bone 17:117S-123S, 1995

14. Morote J, M'Hammed YI, Martinez E, Esquena S, Lorente JA, Gelabert A: Increase of bone alkaline phosphatase after androgen deprivation therapy in patients with prostate cancer. Urology 59:277-280, 2002

15. Park SB, Lee YJ, Chung CK: Bone mineral density changes after ovariectomy in rats as an osteopenic model: stepwise description of double dorso-lateral approach. J Korean Neurosurg Soc 48: 309-312, 2010

16. Proell V, Xu H, Schuler C, Weber K, Hofbauer LC, Erben RG: Orchiectomy upregulates free soluble RANKL in bone marrow of aged rats. Bone 45:677-681, 2009

17. Ryu SJ, Yoo JS, Eom AY, Koh SB, Choi JW: Roles of alendronate and simvastatin in prevention of bone loss in ovariectomized rats. Toxicol Environ Health Sci 3:114-119, 2011

18. Thompson DD, Simmons HA, Pirie CM, Ke HZ: FDA Guidelines and animal models for osteoporosis. Bone 17:125S-133S, 1995

19. Wang YX, Zhang YF, Griffith JF, Zhou H, Yeung DK, Kwok TC, et al: Vertebral blood perfusion reduction associated with vertebral bone mineral density reduction: a dynamic contrastenhanced MRI study in a rat orchiectomy model. J Magn Reson Imaging 28:1515-1518, 2008 